\title{
Influence of Combination Therapy with Immune Modulators on Anti-TNF Trough Levels and Antibodies in Patients with IBD
}

\author{
Tamara van Schaik, BSc, Jeroen P. W. Maljaars, MD, PhD, Rajiv K. Roopram, BSc, Marthe H. Verwey, MA, \\ Nienke Ipenburg, MA, James C. H. Hardwick, MD, PhD, Roeland A. Veenendaal, MD, PhD, \\ and Andrea E. van der Meulen-de Jong, MD, PhD
}

\begin{abstract}
Background: It is important to identify factors that can reduce the incidence of immunogenicity against anti-tumor necrosis factor medication in patients with inflammatory bowel disease. The objective of our study was to evaluate the influence of cotreatment with immune modulators (IMs) on trough levels (TLs) and antidrug antibodies.
\end{abstract}

Methods: The records of all patients with inflammatory bowel disease at the Leiden University Medical Center who received either adalimumab or infliximab (IFX) in the year 2011 and/or 2012 ( $\mathrm{n}=352$ ) were retrospectively evaluated about the assessment of TL and antibodies and use of IM.

Results: Two hundred seventeen patients were included (108 patients IFX; 109 patients adalimumab). Mean TL in the IFX group was higher in the combination therapy group compared with the monotherapy group, 4.6 versus $7.5 \mu \mathrm{g} / \mathrm{mL}, P=0.04$. In the adalimumab group, the difference was not significant. In patients with IFX monotherapy, the incidence of antibody formation was higher compared with patients with combination therapy ( $29.8 \%$ versus $5.7 \%, P=0.001)$. IFX patients with a suboptimal dose of IM had a higher TL compared with patients who had an optimal dose, $P=0.02$. The incidence of antibody formation was lower in IFX patients who immediately started with IMs compared with patients who did not (33.3\% versus $66.7 \%, P=0.04)$.

Conclusions: The influence of combination therapy with IM on TL and antibodies to anti-tumor necrosis factor medication was significant for IFX-treated patients. Patients who started combination therapy immediately developed antibodies less often than patients who started later with concomitant medication.

(Inflamm Bowel Dis 2014;20:2292-2298)

Key Words: anti-TNF- $\alpha$ therapy, trough levels, antibodies, immune modulators, combination therapy

$I^{n+0}$ patients with inflammatory bowel disease, biologicals, such as infliximab (IFX) and adalimumab (ADA), are part of the standard treatment regimen. ${ }^{1-3}$ IFX is a chimeric and ADA is a fully humanized monoclonal immunoglobulin G1 antibody against tumor necrosis factor (TNF) that specifically bind to TNF- $\alpha .{ }^{4,5}$ Several randomized controlled trials have demonstrated the effectiveness of these biologicals as compared with placebo treatment. ${ }^{3,4,6-8}$

Treatment with anti-TNF agents can result in immunogenicity and the formation of antidrug antibodies against these agents. ${ }^{9}$ Antibody formation has been associated with a shorter duration of clinical response, ${ }^{9}$ lower trough levels (TLs) of anti-TNF, ${ }^{7}$ and a higher incidence of infusion reactions. ${ }^{9,10}$ Still, the importance of antibody formation is disputed because other factors than anti-TNF- $\alpha$ antibodies could influence TLs and loss of response, such as variability in the rate of elimination (clearance). ${ }^{11}$ Besides, some studies have not

Received for publication June 11, 2014; Accepted July 31, 2014.

From the Department of Gastroenterology, Leiden University Medical Center, Leiden, the Netherlands.

The authors have no conflicts of interest to disclose.

Reprints: Andrea E. van der Meulen-de Jong, MD, PhD, Department of Gastroenterology and Hepatology, C4P, Leiden University Medical Centre, Albinusdreef 2, 2333 ZA Leiden, the Netherlands (e-mail: ae.meulen@lumc.nl).

Copyright (C) 2014 Crohn's \& Colitis Foundation of America, Inc.

DOI 10.1097/MIB.0000000000000208

Published online 16 September 2014. shown differences between antibody-positive and antibody-negative patients regarding rates of remission, response, endoscopic improvement, C-reactive protein, and colectomy. ${ }^{10,12,13}$ However, detectable serum IFX concentrations have consistently been associated with clinical remission and endoscopic improvements, and an undetectable trough serum IFX level predicts an increased risk for colectomy. ${ }^{13}$

A universally used strategy to reduce antibody formation is maintenance treatment instead of episodic treatment with IFX. ${ }^{10,13}$ Another strategy to reduce antibody formation is to combine antiTNF treatment with immune modulators (IMs). ${ }^{9,10}$ Furthermore, early combined immunosuppression (IFX + azathioprine) is also more effective for induction of remission and reduction of corticosteroid use compared with conventional step-up therapy. ${ }^{14}$ However, the data on the importance of antibody formation and the efficacy of cotreatment with IM to reduce this phenomenon have predominantly been performed on IFX: the influence of combination therapy with IM on TLs and antibodies in patients treated with ADA has been poorly investigated. ${ }^{15}$

We therefore performed a retrospective study on optimization of anti-TNF treatment using IM, using data from our tertiary referral centre. The objective of our study was, first, to evaluate the influence of cotreatment with IM in patients using anti-TNF on anti-TNF TL and antibodies. Second, because IM are frequently given in suboptimal dose because of due to side-effects, we investigated whether a suboptimal dose influenced TL or antibody formation. 


\section{METHODS}

\section{Study Design and Patients}

A retrospective search of medical records was performed of all patients with inflammatory bowel disease (IBD) who received either ADA or IFX in the year 2011 and/or 2012 at the Leiden University Medical Center. Those patients with 1 or more assessments of anti-TNF TLs or antibodies against anti-TNF testing were included in the study and retrospectively evaluated. All laboratory assessments concerning antidrug antibodies, TLs, and IM metabolites were obtained from the medical record, and all assessments performed during the period January 2010-March 2013 in our cohort were included. Besides these laboratory values, demographic and clinical characteristics were collected from the electronically medical records. Characteristics included gender, date of birth, body weight, diagnosis (Crohn's disease or ulcerative colitis), date of diagnosis, smoking status, anti-TNF use, concomitant medication, date of anti-TNF and IM initiation, medication dose, clinical response, and $\mathrm{C}$-reactive protein values. Patients were considered as being treated with combination therapy with IMs if they were at least treated for a period of 10 weeks before laboratory date or stopped no longer than 4 weeks before laboratory date.

For all TL and antibodies to anti-TNF assessments, the reason for ordering the test, date of testing, date of follow-up, and changes to medication were recorded. TL and antibody concentrations were ordered in patients with IBD as decided by the treating physician. Reasons could be: (1) complaints/loss of response, (2) to titrate the dose actively, (3) for clinical interest, and (4) to control TL after a medication change based on previous TL results (follow-up TL assessment). Complaints/loss of response could consist of clinical worsening of symptoms and/or objective inflammation, the latter proven by elevated C-reactive protein or endoscopic activity. The assessments of clinical interest were in absence of clinical complaints (e.g., building up clinical experience, interest because of possible medication stop in [near] future).

Patients who were lost to follow-up or died between 20112012 and March 2013 were followed up until their last contact moment or date of death.

\section{Serum Testing}

TL and antibodies were performed by Sanquin (Amsterdam, the Netherlands) and reported in microgram per milliliter and $\mathrm{AE}$ per milliliter, respectively. TL was defined as serum concentration immediately before an IFX/ADA infusion. A normal TL for IFX was defined as between 3 and $7 \mu \mathrm{g} / \mathrm{mL}$, a low TL as $<3 \mu \mathrm{g} / \mathrm{mL}$, and a high TL as $>7 \mu \mathrm{g} / \mathrm{mL}$. A normal TL for ADA remains unclear in clinical practice but we used the same ranges as for IFX. Antidrug antibody levels for both IFX and $\mathrm{ADA}$ were reported as either negative $(<12 \mathrm{AE} / \mathrm{mL})$ or positive $(>12 \mathrm{AE} / \mathrm{mL})$.

The serum concentration of azathioprine, mercaptopurine, and thioguanine is determined by measuring the 6-thioguanine level in red blood cells. A normal concentration was defined as being 460 to $900 \mathrm{pmol} / 8 \times 10^{8}$ red blood cells.
Furthermore, the optimal therapeutic dose was calculated based on bodyweight, according to the following: azathioprine 2 to $2.5 \mathrm{mg} / \mathrm{kg}$, 6-mercaptopurine 1 to $1.5 \mathrm{mg} / \mathrm{kg}$, thioguanine $0.3 \mathrm{mg} /$ $\mathrm{kg}$, and methotrexate $\geq 15 \mathrm{mg} / \mathrm{wk}$. When the actual dose was below this weight-based calculated dose, they were deemed to be suboptimal. If 6-thioguanine levels had been measured more than once in the same patient, the first measurement was included in this study.

\section{Statistical Analysis}

All statistical analyses were performed with SPSS (version 20; SPSS, Inc., Chicago, IL). Demographic and baseline characteristics were compared between groups with the use of the chi-squared test for categorical variables and the independent sample t-test for continuous variables. Trough serum levels were compared between the monotherapy group and the combination therapy group by using an independent sample t-test. The proportion of patients with a positive antibody status was compared between the monotherapy group and the combination therapy group by using a chi-squared test. Similarly, in the subgroup analyses on dose and duration of treatment with IM, we compared the 2 groups with the use of a chisquared test and an independent sample t-test.

\section{RESULTS}

\section{Baseline Characteristics}

A total of 352 anti-TNF users in 2011 and/or 2012 with IBD were identified. In 217 patients, either anti-TNF TLs or anti-TNF antibody levels had been measured at least once (TL: $n=211$ and/ or antibodies: $n=203$ ) during the period January 2010-March 2013 (Fig. 1). Baseline demographic and clinical characteristics of the 217 patients are shown in Table 1 for IFX and in Table 2 for ADA. We compared the monotherapy group $(n=128)$ with the

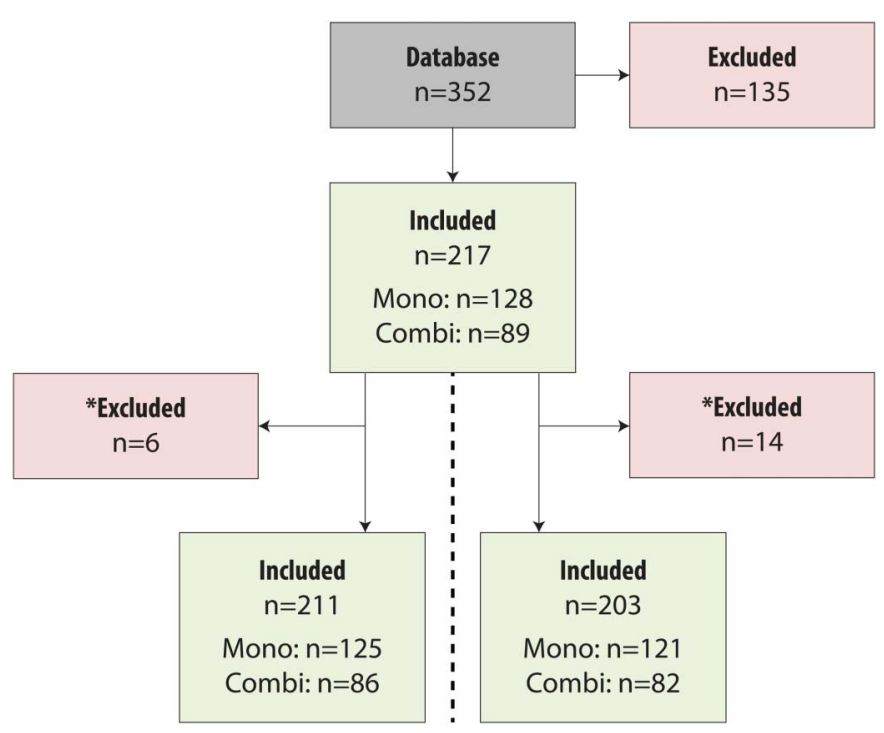

FIGURE 1. Study design and number of patients. AB: number of patients with antibody testing. Mono: Monotherapy group. Combi: Combination therapy group. *Only tested on antibodies. ${ }^{+}$Only tested on TL. 
TABLE 1. Baseline Patient Characteristics for IFX

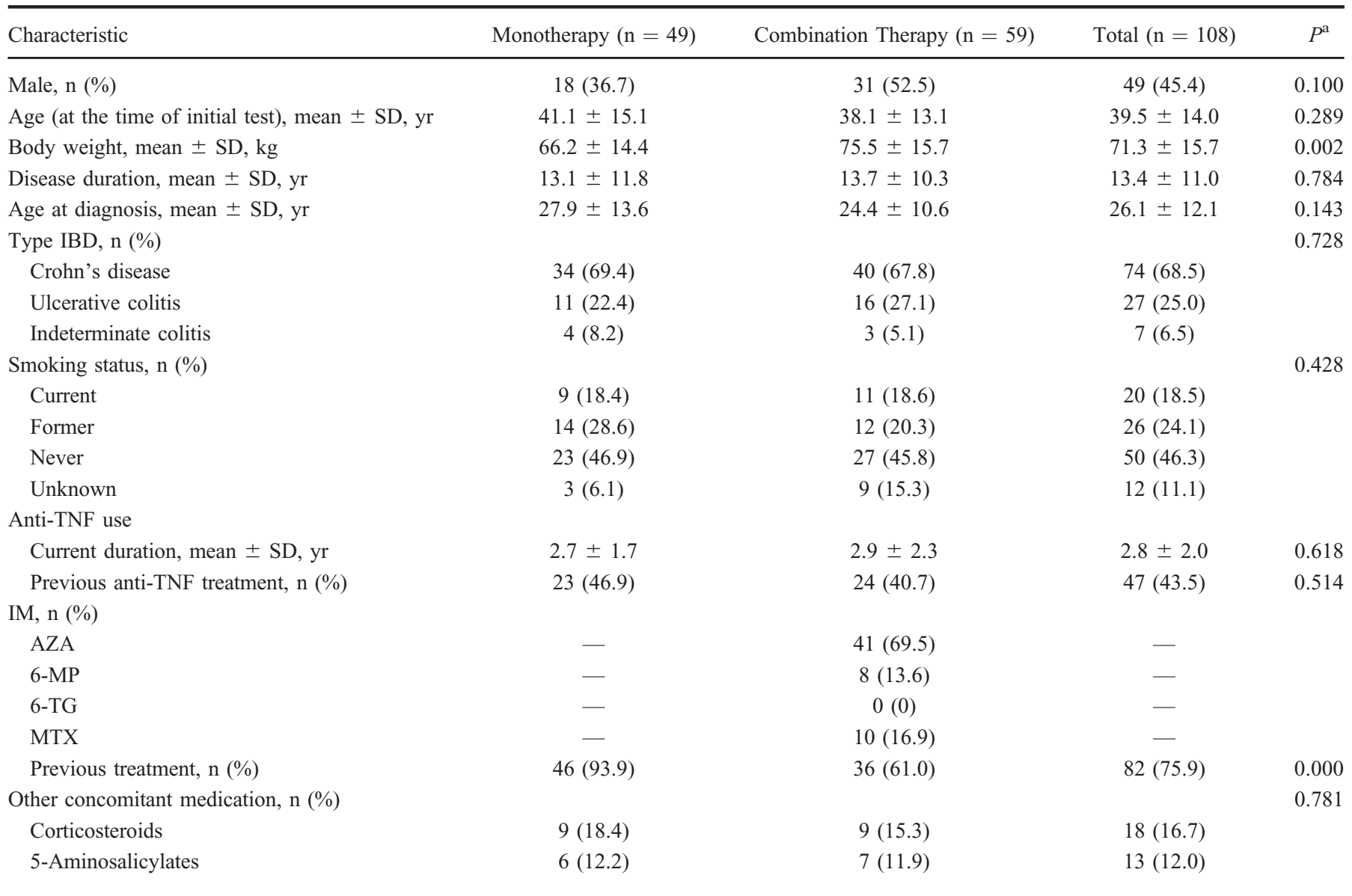

${ }^{\mathrm{a}} P$ values for monotherapy versus combination therepy. $P$ values were calculated with the use of independent sample t-test and chi-squared test.

combination therapy group $(\mathrm{n}=89)$. For IFX patients, body weight and previous treatment with IM differed significantly between the 2 groups. For ADA patients, age at the time of initial test, current duration of anti-TNF use, and previous treatment with IM differed significantly between the 2 groups.

\section{Trough Levels}

In 211 of the 217 patients, at least 1 TL was determined. Of these 211 patients, 102 were treated with IFX and 109 were treated with ADA.

TL in patients treated with IFX was low in 43 patients (42.2\%), normal in 25 patients $(24.5 \%)$, and high in 34 patients $(33.3 \%)$. For patients treated with ADA, these numbers were 10 $(9.2 \%), 26(23.9 \%)$, and $73(67.0 \%)$, respectively. Most of the laboratory TL assessments were requested because of loss of response (62.5\%), followed by clinical interest $(27.6 \%)$ and titration $(9.7 \%)$. However, TL did not differ between the different indications (Fig. 2).

In Figure 3, outcome of TL for all TL patients $(n=211)$, $\operatorname{IFX}(\mathrm{n}=102)$, and ADA $(\mathrm{n}=109)$ are shown. In the IFX group, mean TL was significantly higher in patients treated with combination therapy compared with the patients treated with monotherapy (7.5; $\mathrm{SD}=7.34 \mu \mathrm{g} / \mathrm{mL}$ versus $4.58 ; \mathrm{SD}=6.66 \mu \mathrm{g} / \mathrm{mL}$, $P=0.04)$. No such difference was found in the ADA group $(11.47 ; \mathrm{SD}=8.18 \mu \mathrm{g} / \mathrm{mL}$ versus $13.10 ; \mathrm{SD}=11.97 \mu \mathrm{g} / \mathrm{mL}$, not significant).

\section{Antibody Formation}

Results for antibodies were available for 203 patients (100 IFX and 103 ADA). In patients with IFX, combination therapy ( $\mathrm{n}=47$ ) reduced the incidence of antibody formation compared with patients with monotherapy $(\mathrm{n}=53)$; respectively $(29.8 \%$ versus $5.7 \%, P=0.001$, Fig. 4). No effect of combination therapy was observed in the ADA patients (Fig. 4).

\section{TLs and Antibodies: Effect of Previous IM Use}

Of the 79 ADA monotherapy patients, 32 used IM during their current ADA treatment episode. When comparing TL and antibodies between patients who have previously used an IM 


\section{TABLE 2. Baseline Patient Characteristics for ADA}

\begin{tabular}{|c|c|c|c|c|}
\hline Characteristic & Monotherapy $(\mathrm{n}=79)$ & Combination Therapy $(\mathrm{n}=30)$ & Total $(\mathrm{n}=109)$ & $P^{\mathrm{a}}$ \\
\hline Male, n (\%) & $25(31.6)$ & $14(46.7)$ & $39(35.8)$ & 0.144 \\
\hline Age (at the time of initial test), mean $\pm \mathrm{SD}, \mathrm{yr}$ & $41.6 \pm 12.0$ & $35.7 \pm 12.1$ & $40.0 \pm 12.3$ & 0.027 \\
\hline Body weight, mean $\pm \mathrm{SD}, \mathrm{kg}$ & $72.4 \pm 19.2$ & $67.2 \pm 15.4$ & $71.0 \pm 18.3$ & 0.145 \\
\hline Disease duration, mean $\pm \mathrm{SD}$, yr & $16.0 \pm 10.3$ & $12.7 \pm 8.7$ & $15.1 \pm 10.0$ & 0.096 \\
\hline Age at diagnosis, mean $\pm \mathrm{SD}$, yr & $25.6 \pm 9.3$ & $23.0 \pm 7.4$ & $24.9 \pm 8.9$ & 0.143 \\
\hline Ulcerative colitis & $5(6.3)$ & $1(3.3)$ & $6(5.5)$ & \\
\hline Indeterminate colitis & $4(5.1)$ & $0(0)$ & $4(3.7)$ & \\
\hline Smoking status, n (\%) & & & & 0.837 \\
\hline Current & $16(20.3)$ & $5(16.7)$ & $21(19.3)$ & \\
\hline Former & $20(25.3)$ & $6(20.0)$ & $26(23.9)$ & \\
\hline Current duration, mean $\pm \mathrm{SD}$, yr & $2.6 \pm 1.4$ & $1.8 \pm 1.1$ & $2.4 \pm 1.4$ & 0.005 \\
\hline Previous anti-TNF treatment, $\mathrm{n}(\%)$ & $52(65.8)$ & $22(73.3)$ & $74(67.9)$ & 0.453 \\
\hline IM, n (\%) & - & & - & \\
\hline AZA & - & $18(60.0)$ & - & \\
\hline 6-MP & - & $7(23.3)$ & - & \\
\hline $6-\mathrm{TG}$ & - & $0(0)$ & - & \\
\hline MTX & - & $5(16.7)$ & - & \\
\hline Previous treatment, $\mathrm{n}(\%)$ & $72(91.1)$ & $18(60.0)$ & $90(82.6)$ & 0.000 \\
\hline Other concomitant medication, $\mathrm{n}(\%)$ & & & & 0.640 \\
\hline Corticosteroids & $6(7.6)$ & $4(13.3)$ & $10(9.2)$ & \\
\hline 5-aminosalicylates & $2(2.5)$ & $1(3.3)$ & $3(2.8)$ & \\
\hline
\end{tabular}

$\operatorname{IFX}(n=102)$

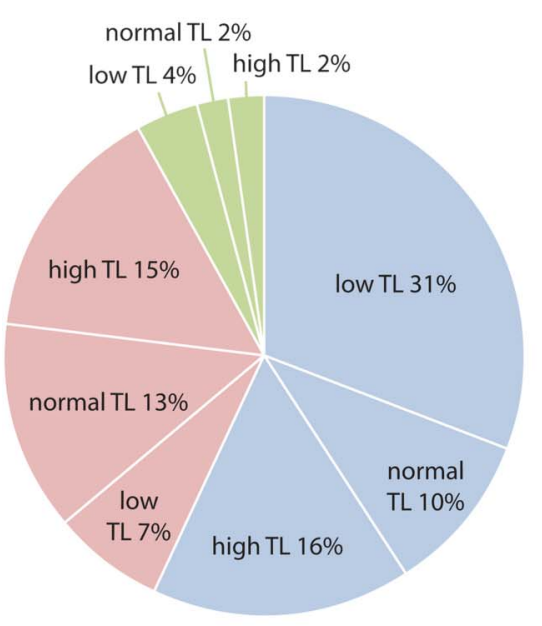

ILR

Clinical interest

Titration

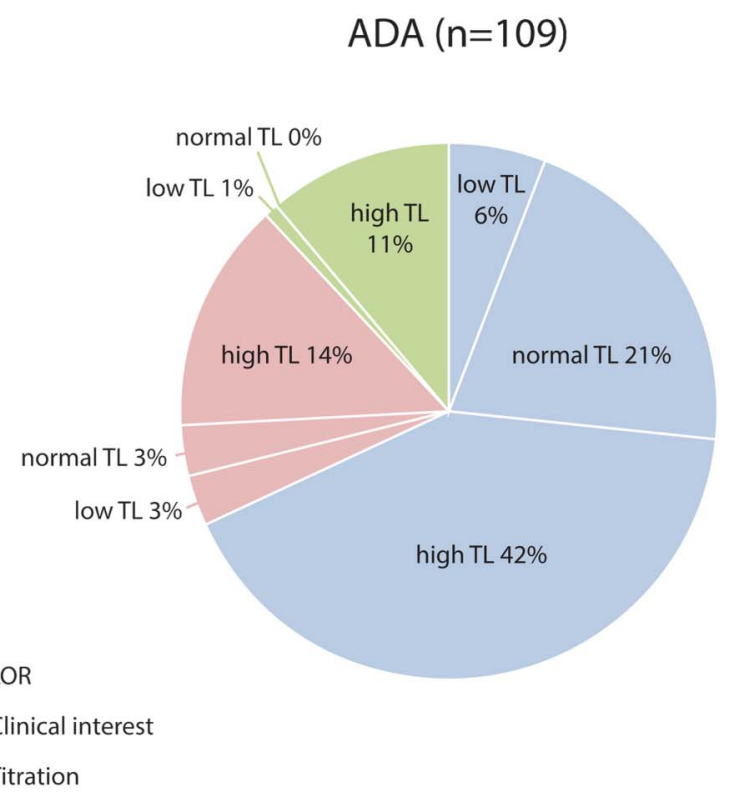

FIGURE 2. Indications for laboratory assessments with outcomes in IFX and ADA patients. LOR: Loss of response. 


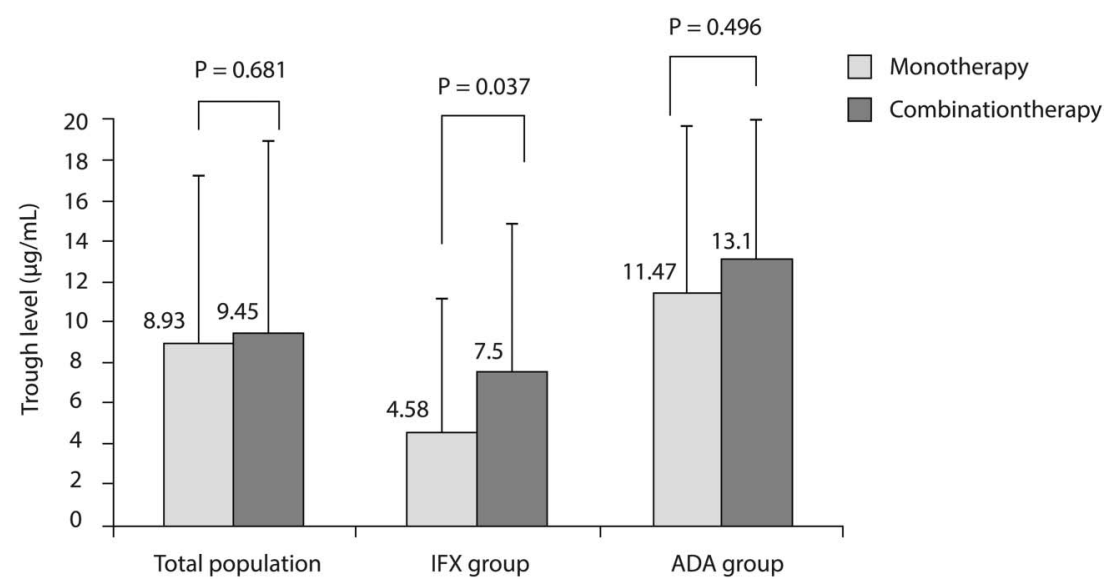

FIGURE 3. Mean TL in patients with IBD in the total population and in a subanalysis for IFX and ADA. The numbers above the bars correspond to the mean TL. The error bars correspond to the SD.

during the current ADA episode (but not at the time of TL and/or antibody status assessment) and IM nonusers, no differences were observed in TLs (9.42; $\mathrm{SD}=6.25 \mu \mathrm{g} / \mathrm{mL}$ versus 12.73 ; $\mathrm{SD}=$ $9.0 \mu \mathrm{g} / \mathrm{mL}$, respectively) and formation of antibodies $(16.7 \%$ versus $23.7 \%$, respectively, $P=0.477$ ).

Similarly, in IFX patients, no difference was observed in TLs (4.92; $\mathrm{SD}=7.4 \mu \mathrm{g} / \mathrm{mL}$ versus $4.85 ; \mathrm{SD}=5.9 \mu \mathrm{g} / \mathrm{mL}$, $P=0.975)$ and antibody formation (17.6\% versus $29.6 \%$, respectively, $P=0.371$ ) between previous IM users, earlier during the use of the current anti-TNF agent and IM nonusers.

\section{Effect of IM Dose on TLs and Antibody Formation}

Of the 211 patients with known TL, 86 patients received combination therapy with IMs at the time of TL assessment (Fig. 1). IFX-patients with a suboptimal IM dose had a higher TL compared with patients who were optimally dosed $(9.81 ; \mathrm{SD}=8.08 \mu \mathrm{g} / \mathrm{mL}$ versus 5.36; $\mathrm{SD}=5.95 \mu \mathrm{g} / \mathrm{mL} ; P=0.024)$, whereas for ADA, no such difference was observed (data not shown).

In 82 patients who received combination therapy $(n=89)$, antibodies were measured (Fig. 1). The dosing of the IM did not influence the incidence of antibody formation for patients using IFX ( $\mathrm{n}=53$; suboptimal dosed IM versus optimal dosed IM; $8.0 \%$ versus $3.6 \%$, not significant) nor for patients using ADA ([n $=29] ; 15.4 \%$ versus $18.75 \%$, respectively; not significant). The same was the case in patients with known 6-thioguanine serum levels (5\% versus $11 \%$, not significant).

\section{Timing of IM Therapy}

We compared TL and formation of antibodies between patients who started IM immediately after starting anti-TNF medication and those who started later. No difference was observed for both IFX (7.85; SD = $7.87 \mu \mathrm{g} / \mathrm{mL}$ versus 6.35 ; $\mathrm{SD}=5.33 \mu \mathrm{g} / \mathrm{mL})$ and $\mathrm{ADA}(13.89 ; \mathrm{SD}=11.46 \mu \mathrm{g} / \mathrm{mL}$ versus

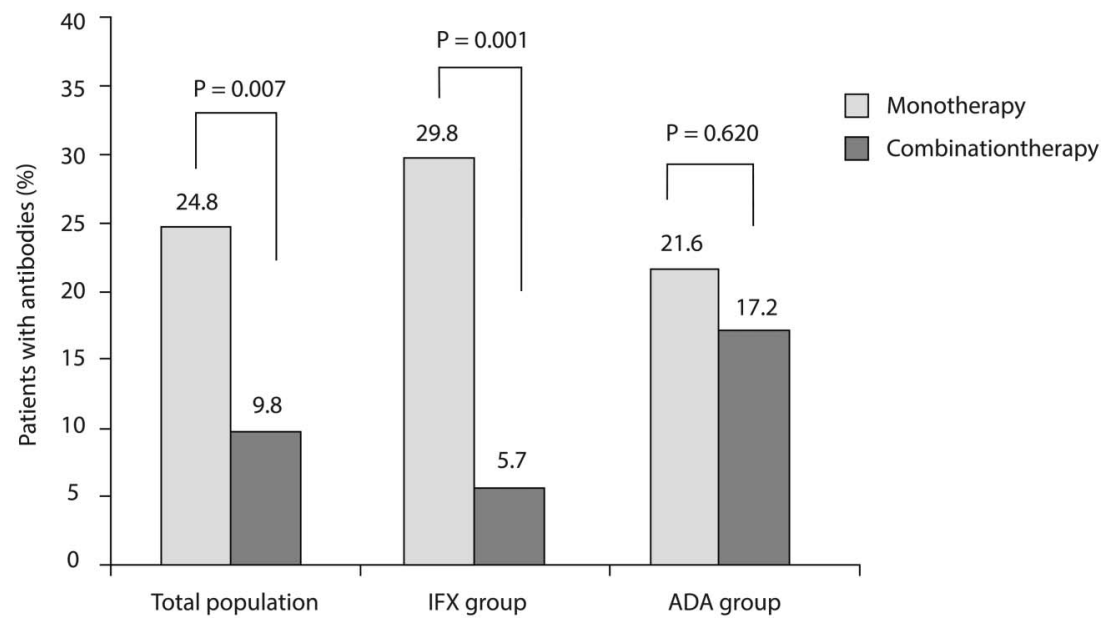

FIGURE 4. Incidence of antibodies to anti-TNF in monotherapy versus combination therapy in the total population and in a subanalysis for IFX and ADA. The numbers above the bars correspond to the percentage antibodies. 
11.72; SD $=13.25 \mu \mathrm{g} / \mathrm{mL})$. Regarding IFX, the incidence of antibody formation was lower in patients who started IM immediately compared with patients who did not ( $[\mathrm{n}=53] ; 2.4 \%$ versus $18.2 \%$, respectively, $P=0.044)$. This relationship was not observed in patients using ADA ([n $=29]$; 30.0\% versus $10.5 \%$, respectively, not significant).

A total of 128 patients did not use IM at the time of TL assessment. Ten patients did not receive any previous IM treatment. In the remaining 118 patients, the main reason for stopping IM previously was "intolerance" to the agent (20.4\%), which could consist of nausea, vomiting, headaches, dizziness, tiredness, sleeplessness, or general malaise. Other reasons were liver function test abnormalities and pancreatitis (12.6\%), infection (12.0\%), stepdown therapy $(12.0 \%)$, patient preference $(11.0 \%)$, dermatological side-effects $(6.5 \%)$, leucopenia/anemia/thrombocytopenia $(5.8 \%)$, low efficacy $(5.8 \%)$, miscellaneous $(5.2 \%)$, joint and muscle complaints (3.6\%), and unknown (5.2\%).

\section{DISCUSSION}

In this retrospective cohort of 217 patients, we evaluated the influence of combination therapy with IM on TL and antibodies to anti-TNF medication in patients with IBD. From this study, we can draw a number of interesting conclusions:

First, in patients receiving ADA, no beneficial effect was observed of IM comedication regarding TLs and antibody formation. This is in contrast to patients with IFX, where combination therapy led to higher TLs and a lower presence of antibodies.

Second, in IFX patients receiving IM comedication, the dose of the IM does not influence TLs or antibody formation.

Third, starting comedication together with IFX seems to be beneficial, as these patients developed antibodies at a lower rate than patients who started comedication later.

Presence of antidrug antibodies has been shown to reduce the clinical efficacy of ADA. ${ }^{16}$ However, whether IM comedication prevents formation of anti-ADA antibodies is uncertain. For instance, Karmiris et $\mathrm{al}^{17}$ retrospectively studied 169 ADA patients, of whom 62 used IM. In this study, IM comedication did not influence development of antidrug antibodies or TL. ${ }^{17}$ In contrast, in the prospective CLASSIC-II study, antibodies were only seen in patients who were on ADA monotherapy, which would suggest a preventive effect of IM. ${ }^{8}$ In our study in 109 patients using ADA for an average period of over 2 years, no effect was observed of using IM comedication on antibody formation or TLs. A large part of the patients on monotherapy was on combination therapy before the assessment of TL and antibodies. To rule out that previous IM use had a protective effect on TL and antibody formation, we compared monotherapy patients who had and who had not used IM during the current ADA period. No effect of previous IM use was observed on TL and antibody formation.

One factor complicating the interpretation of this and other studies is that presence of antibodies may be intermittent; patients who are antibody-positive can convert back to antibody-negative status. ${ }^{10}$ This may have affected results in the above-mentioned articles and in our study. Another factor that could complicate the interpretation is the fact that most anti-TNF monotherapy patients have used an IM in the past (93.9\% for IFX and 65.8\% for ADA, Tables 1 and 2) and also during current anti-TNF use $(63 \%$ for IFX and $45.1 \%$ for ADA).

In our study, we did not assess clinical response, and IM comedication may improve clinical response, as was suggested in a meta-analysis ${ }^{18}$ presented at the 2014 ECCO meeting. However, this meta-analysis used primarily post-hoc data of prospective studies and data from retrospective studies, which means that these results should be interpreted with some caution. Because no prospective studies have been performed that specifically assess the benefit of IM comedication in patients using ADA, our and other ${ }^{17,19}$ data suggest that there is no indication for standard combination therapy in patients starting ADA.

In clinical practice, many patients are unable or unwilling to tolerate the optimal weight-based dose of azathioprine or 6-mercaptopurine. The effects of IM use in patients using a suboptimal dose on TL and antibody formation have, to our knowledge, not yet been described. Surprisingly, IFX patients who used a suboptimally (based on weight) dosed IM had a significantly higher TL than patients who were optimal dosed. No effect of suboptimal dosing on antibody formation was observed in IFX patients. These data demonstrate that in patients who were unable or unwilling to tolerate the full dose of IM, a beneficial effect of a lower IM dose may still be expected.

Cotreatment of IFX and an IM is standard practice in many centers, and the benefits of IM cotreatment have been previously demonstrated. Our study shows that IFX patients who immediately started with IM had a lower incidence of antibodies compared with patients who started later with IM. This indicates that early combined immunosuppression is better than step-up therapy.

In conclusion, cotreatment with an IM seems beneficial for patients on IFX and should be started simultaneously with the start of IFX. In patients who were unable or unwilling to tolerate the full dose of IM, a beneficial effect of a lower IM dose was still observed.

In contrast, no benefit of IM cotreatment was observed in the ADA-treated patients. Given the results of this and other studies, there is no indication for standard IM comedication in patients using ADA.

\section{REFERENCES}

1. Baumgart DC, Sandborn WJ. Inflammatory bowel disease: clinical aspects and established and evolving therapies. Lancet. 2007;369:1641-1657.

2. Kornbluth A, Sachar DB. Ulcerative colitis practice guidelines in adults (update): American College of Gastroenterology, Practice Parameters Committee. Am J Gastroenterol. 2004;99:1371-1385.

3. Rutgeerts P, Sandborn WJ, Feagan BG, et al. Infliximab for induction and maintenance therapy for ulcerative colitis. $N$ Engl J Med. 2005;353: $2462-2476$

4. Colombel JF, Sandborn WJ, Rutgeerts P, et al. Adalimumab for maintenance of clinical response and remission in patients with Crohn's disease: the CHARM trial. Gastroenterology. 2007;132:52-65.

5. Targan SR, Hanauer SB, van Deventer SJ, et al. A short-term study of chimeric monoclonal antibody cA2 to tumor necrosis factor alpha for Crohn's disease. Crohn's Disease cA2 Study Group. $N$ Engl J Med. 1997;337:1029-1035. 
6. Hanauer SB, Feagan BG, Lichtenstein GR, et al. Maintenance infliximab for Crohn's disease: the ACCENT I randomised trial. Lancet. 2002;359: 1541-1549.

7. Hanauer SB, Sandborn WJ, Rutgeerts $P$, et al. Human anti-tumor necrosis factor monoclonal antibody (adalimumab) in Crohn's disease: the CLASSIC-I trial. Gastroenterology. 2006;130:323-333; quiz 591.

8. Sandborn WJ, Hanauer SB, Rutgeerts P, et al. Adalimumab for maintenance treatment of Crohn's disease: results of the CLASSIC II trial. Gut. 2007;56:1232-1239.

9. Baert F, Noman M, Vermeire S, et al. Influence of immunogenicity on the long-term efficacy of infliximab in Crohn's disease. N Engl J Med. 2003; 348:601-608.

10. Hanauer SB, Wagner CL, Bala M, et al. Incidence and importance of antibody responses to infliximab after maintenance or episodic treatment in Crohn's disease. Clin Gastroenterol Hepatol. 2004;2:542-553.

11. St Clair EW, Wagner CL, Fasanmade AA, et al. The relationship of serum infliximab concentrations to clinical improvement in rheumatoid arthritis: results from ATTRACT, a multicenter, randomized, double-blind, placebo-controlled trial. Arthritis Rheum. 2002;46:1451-1459.

12. Seow $\mathrm{CH}$, Newman $\mathrm{A}$, Irwin $\mathrm{SP}$, et al. Trough serum infliximab: a predictive factor of clinical outcome for infliximab treatment in acute ulcerative colitis. Gut. 2010;59:49-54
13. Maser EA, Villela R, Silverberg MS, et al. Association of trough serum infliximab to clinical outcome after scheduled maintenance treatment for Crohn's disease. Clin Gastroenterol Hepatol. 2006;4: $1248-1254$

14. D'Haens G, Baert F, van Assche G, et al. Early combined immunosuppression or conventional management in patients with newly diagnosed Crohn's disease: an open randomised trial. Lancet. 2008;371:660-667.

15. Sandborn WJ. State-of-the-art: immunosuppression and biologic therapy. Dig Dis. 2010;28:536-542.

16. West RL, Zelinkova Z, Wolbink GJ, et al. Immunogenicity negatively influences the outcome of adalimumab treatment in Crohn's disease. Aliment Pharmacol Ther. 2008;28:1122-1126.

17. Karmiris K, Paintaud G, Noman M, et al. Influence of trough serum levels and immunogenicity on long-term outcome of adalimumab therapy in Crohn's disease. Gastroenterology. 2009;137:1628-1640.

18. Kopylov U, Al-Taweel T, Yaghoobi M, et al. Adalimumab monotherapy versus combination therapy with adalimumab and immunomodulators for Crohn's disease: A meta-analysis. [published online ahead of print July 24, 2014]. doi: 10.1016/j.crohns.2014.07.003.

19. Vermeire S, Colombel J, Robinson AM, et al. Effect of concomitant immunomodulator use on the efficacy of adalimumab in Crohn's disease patients stratified by prior anti-TNF use. ECCO 2011 Abstract P.137. 